\title{
Vulval angiomyofibroblastoma
}

\author{
H D P Atapattu ${ }^{1}$, C S P Sosai ${ }^{2}$ \\ Sri Lanka Journal of Obstetrics and Gynaecology 2011; 33: 168-169
}

\begin{abstract}
A 25-year old woman presented to the Gynaecology Clinic at Base Hospital, Diyatalawa with a history of a lump in the right labium majus for 3 month duration. The lump was surgically excised. The histopathological diagnosis was an angiomyofibroblastoma (AMFB) a rare benign mesenchymal tumour arising in the genital region predominantly of the pre-menopausal women.
\end{abstract}

Key words: angiomyofibroblastoma, benign vulval tumours.

\section{Introduction}

Angiomyofibroblastoma is a rare benign mesenchymal tumour which occurs in the genital region, especially the superficial areas of vulva, of premenopausal women ${ }^{1,2}$. It was first described by Fletcher et al in $1992^{3}$. AMFB can rarely occur in the spermatic cord and the scrotum in men ${ }^{4}$. Since 1992 approximately 150 cases have been documented in the literature ${ }^{5}$. The tumour has an extremely low risk of recurrence if completely excised ${ }^{6}$.

\section{Case report}

A 25-year-old woman presented to the Gynaecology Clinic at Base Hospital, Diyatalawa complaining of a lump at vulva for 3 months. It was gradually enlarging but was not painful and was not ulcerated. She has had a vaginal birth two months back.

On examination, there was a lump in the right labium majus. It was $6 \mathrm{~cm}$ in diameter with an irregular surface, firm, non-tender, non-fluctuant and was not attached to the underlying structures. The overlying skin was normal and there were no abnormal blood vessels. The regional lymph nodes were not enlarged. There was no cough impulse.

\footnotetext{
${ }^{1}$ Consultant Obstetrician and Gynaecologist, Base Hospital Embilipitiya.

${ }^{2}$ Consultant Pathologist, GH Badulla.

Correspondence: Harsha Atapattu

E-mail: hatapattu@yahoo.co.uk
}

The lump was surgically excised. It resembled a fibroid on macroscopic appearance. The pathological diagnosis was an angiomyofibroblastoma.

\section{Discussion}

Angiomyofibroblastoma originates from an immature multipotent cell of the connective tissue situated around the vessels, which is capable of differentiating into similar tumoral varieties ${ }^{7}$. Clinically AMFB presents as slow-growing, painless lumps with a low tendency for local recurrence, and usually are misdiagnosed as Bartholin's gland cysts $^{8,9}$. They are mostly well-circumscribed masses with a rubbery texture, measuring $0.5-12 \mathrm{~cm}$ in diameter ${ }^{7,9}$.

AMFB is histologically characterized by alternating hypercellular and hypocelluar areas with an abundance of venular or capillary-sized blood vessels. Tumour cells are spindled, plump spindled or plasmacytoid stromal cells that cluster around blood vessels within an oedematous to collagenous matrix, sometimes forming solid compact foci. Nuclei are bland. Mitotic figures are absent or very sparse $\mathrm{s}^{2,8}$.

Immunohistochemical staining is diagnostic in $\mathrm{AFMB}^{10}$. Immunohistochemically the stromal cells are reactive for vimentin and desmin, but not cytokeratin, muscle-specific actin, alpha-smooth muscles actin or S-100 protein. Both oestrogen and progesterone receptors are diffusely expressed in the stromal cells, suggesting the sex-steroid dependency of the tumour ${ }^{2,8,9}$.

The tumours may rarely be associated with a malignant counterpart (angiomyofibrosarcoma) ${ }^{10}$.

The differential diagnosis includes

1. Aggressive angiomyxoma

2. Cellular angiofibroma

3. Endothelial stromal sarcoma ${ }^{6}$.

Other uncommon sites of occurrence such as the vaginal vault and fallopian tubes have rarely been reported $^{11,12,13}$. 


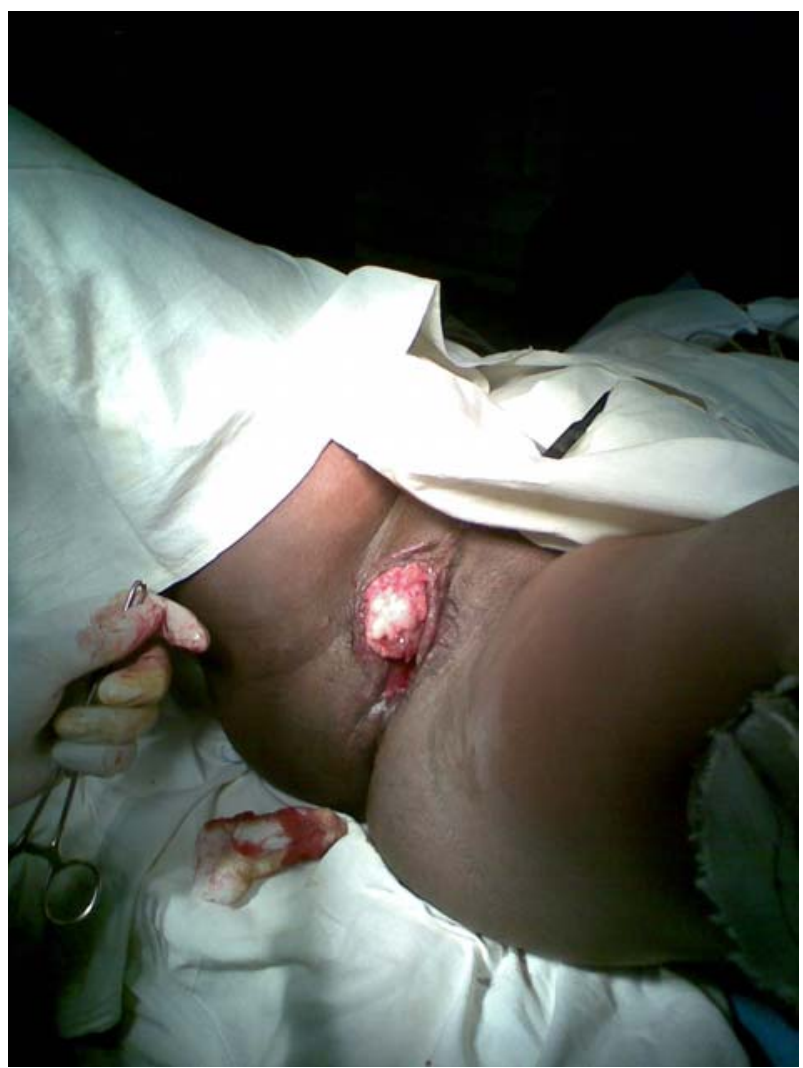

\section{References}

1. Hsu IH, Chang TC, Wu CT, Chen RJ, Chow SN. Angiomyofibroblastoma of the vulva. J Formos Med Assoc 2004; 103: 467-71.

2. Omori M, Toyoda H, Hirai T, Ogino T, Okada S. Angiomyofibroblastoma of the vulva: a large pedunculated mass formation. Acta Med Okayama 2006; 60: 237-42.

3. Fletcher CD, Tsang WY, Fisher C, Lee KC, Chan JK. Angiomyofibroblastoma of the vulva. A benign neoplasm distinct from aggressive angiomyxoma. Am J Surg Pathol 1992; 16: 372-82.

4. Laskin WB, et al. Angiomyofibroblastoma-like tumor of the male genital tract: analysis of 11 cases with comparisons to female angiofibroblastoma and spindle cell lipoma. Am J Surg Pathol 1998; 22(1): 6-16.

5. McCormick M, Riley S. Polypoid Angiomyofibroblastoma: Case Report and Review of the Literature. WMJ 2002; 101(5): 44-6.

6. Chakravarty-Vartak SU, Dhurde AV, Vartak SS, Badarkhe G, Veer S. Vaginal angiomyofibroblastoma. Bombay Hospital Journal 2008; 50(1): 96-8.

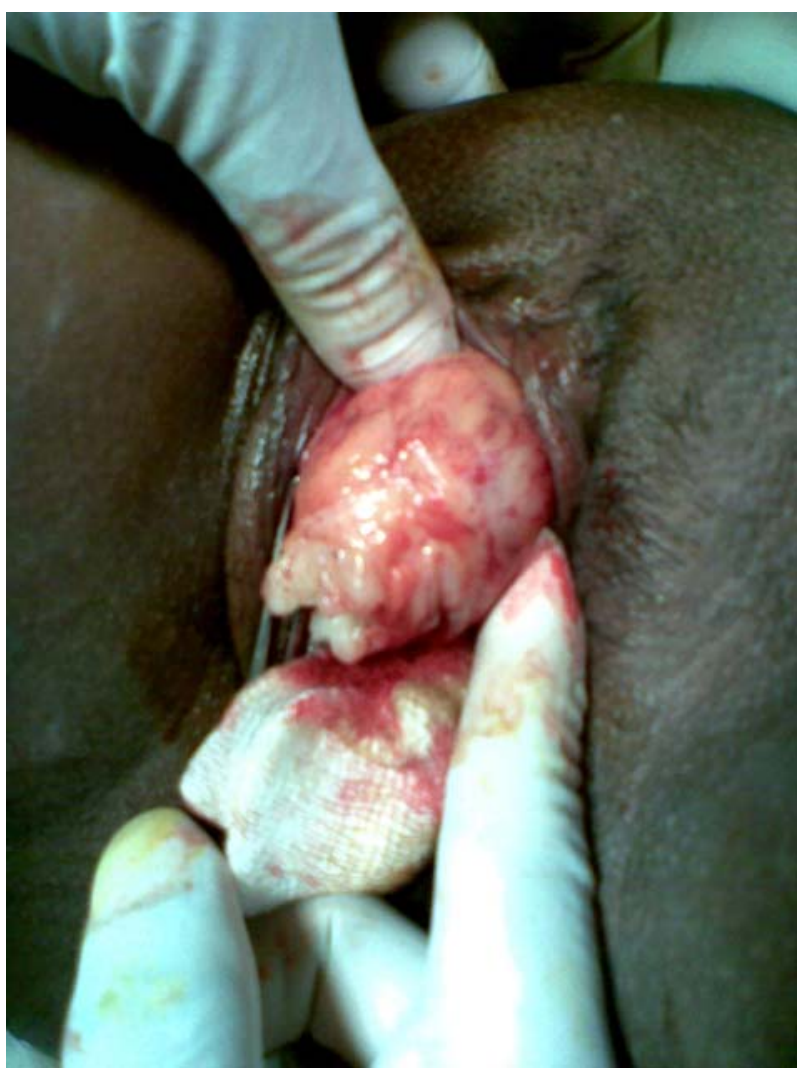

7. Indraccolo U, Sanctis E D, Liberati F, Ippolito M, Indraccolo S N. Giant angiomyofibroblastoma of the vulva. Scot Med J 2008; 53(3): 57.

8. BaratS, Tirgar-Tabari S, ShafaeeS. Angiomyofibroblastoma of the Vulva. Archives of Iranian Medicine 2008; 11: 224-6.

9. Fletcher CD, Tsang WY, Fisher C, Lee KC, Chan JK. Angiomyofibroblastoma of the vulva. A benign neoplasm distinct from aggressive angiomyxoma. Am J Surg Pathol 1993; 17(4): 423-4.

10. Faraj R, Sunder M, Sooltan A R, Kumari S, Golka D, Steel M. Angiomyofibroblastoma of the vagina. J Obstet Gynaecol Can 2007; 29(5): 399.

11. Barboza O, Flores J, et al. Angiomyofibroblastoma of the Vagina. Rev Inst Nal Cancerol (Mex) 2000; 46(4): 247-50.

12. Nielsen G, Rosenberg A, et al. Angiomyofibroblastoma of the vulva and vagina. Mod Pathol 1996; 9(3): 284-91.

13. Nielsen G, et al. Mesenchymal tumors and tumor-like lesions of the female genital tract: a selective review with emphasis on recently described entities. Int J Gynecol Pathol 2001; 20(2): 105-27. 